\title{
Conformational Properties of the SDS-bound State of $\alpha$-Synuclein Probed by Limited Proteolysis: Unexpected Rigidity of the Acidic C-Terminal Tail
}

\author{
Patrizia Polverino de Laureto ${ }^{1}$, Laura Tosatto ${ }^{1}$, Erica Frare ${ }^{1}$, Oriano Marin ${ }^{1}$, \\ Vladimir N. Uversky ${ }^{2}$ and Angelo Fontana ${ }^{1}$ \\ ${ }^{1}$ CRIBI Biotechnology Centre, University of Padua, Viale G. Colombo 3, 35121 Padua, Italy, \\ ${ }^{2}$ Department of Biochemistry and Molecular Biology, Medical School, Indiana University, \\ Indianapolis, IN, USA, and Molecular Kinetics Inc., Indianapolis, IN, USA
}

\section{SUPPORTING INFORMATION}

TABLE S1: Molecular masses of peptide fragments of $\alpha$-syn digested with thermolysin in the absence of SDS.

FIGURE S1: RP-HPLC analysis of $\alpha$-syn digested in the presence of SDS by repeated additions of thermolysin.

FIGURE S2: RP-HPLC analysis of the proteolysis mixture of fragment 108-140 digested with thermolysin and of the fingerprinting mixture of fragment 112-140 digested with V8protease.

TABLE S2: Molecular masses of peptides obtained by digesting fragment 112-140 with V8protease.

FIGURE S3: ESI-MS of fragment 112-140 in the absence or presence of calcium ions. 
Table S1: Analytical Characterization of Fragments Obtained by Proteolysis of $\alpha$-Synuclein with Thermolysin in the Absence of SDS.

\begin{tabular}{|c|c|c|c|}
\hline \multirow[b]{2}{*}{$\mathrm{RT}(\min )^{a}$} & \multicolumn{2}{|c|}{ Molecular mass (Da) } & \multirow[b]{2}{*}{ fragment } \\
\hline & observed $^{b}$ & calculated $^{c}$ & \\
\hline 10.6 & 1045.5 & 1046.1 & $77-87$ \\
\hline 11.4 & 1196.7 & 1197.3 & $40-51$ \\
\hline 11.4 & 1227.6 & 1228.2 & $100-111$ \\
\hline 11.7 & 607.9 & 607.6 & $136-140$ \\
\hline 12.3 & 1814.8 & 1815.1 & $8-25$ \\
\hline 12.7 & 1012.1 & 1012.0 & $124-132$ \\
\hline 13.2 & 2614.5 & 2614.9 & $26-51$ \\
\hline 13.4 & 1434.8 & 1435.6 & $26-39$ \\
\hline 13.7 & 1385.9 & 1386.4 & $112-123$ \\
\hline 14.5 & 827.5 & 827.0 & $1-7$ \\
\hline 14.8 & 3232.6 & 3232.7 & $8-39$ \\
\hline 14.8 & 2380.1 & 2380.5 & $112-132$ \\
\hline 14.8 & 2785.9 & 2786.9 & $112-135$ \\
\hline 15.3 & 3376.0 & 3376.5 & $112-140$ \\
\hline 15.5 & 510.2 & 510.8 & $1-4$ \\
\hline 17.2 & 4412.8 & 4412.0 & $8-51$ \\
\hline 17.8 & 5184.5 & 5185.5 & $95-140$ \\
\hline 18.5 & 4589.8 & 4586.8 & $100-140$ \\
\hline 19.5 & 5818.7 & 5817.2 & $88-140$ \\
\hline 21.4 & 6845.2 & 6845.3 & $77-140$ \\
\hline 28.2 & 3457.1 & 3457.8 & $52-87$ \\
\hline 30.3 & 9257.9 & 9257.1 & $52-140$ \\
\hline 35.7 & 14462.7 & 11460.1 & $1-140$ \\
\hline
\end{tabular}

${ }^{a}$ Proteolysis of $\alpha$-syn with thermolysin was conducted in Tris- $\mathrm{HCl}$ buffer, $\mathrm{pH} 7.5$, in the absence of SDS. An aliquot of the proteolysis mixture was analyzed by RP-HPLC and the peptide material eluted from the reverse-phase C18 column analyzed for molecular masses by ESI-MS (see Figure 3B). ${ }^{b}$ Determined by ESI-MS. ${ }^{c}$ Molecular masses calculated on the basis of the amino acid sequence of human $\alpha$-syn. 


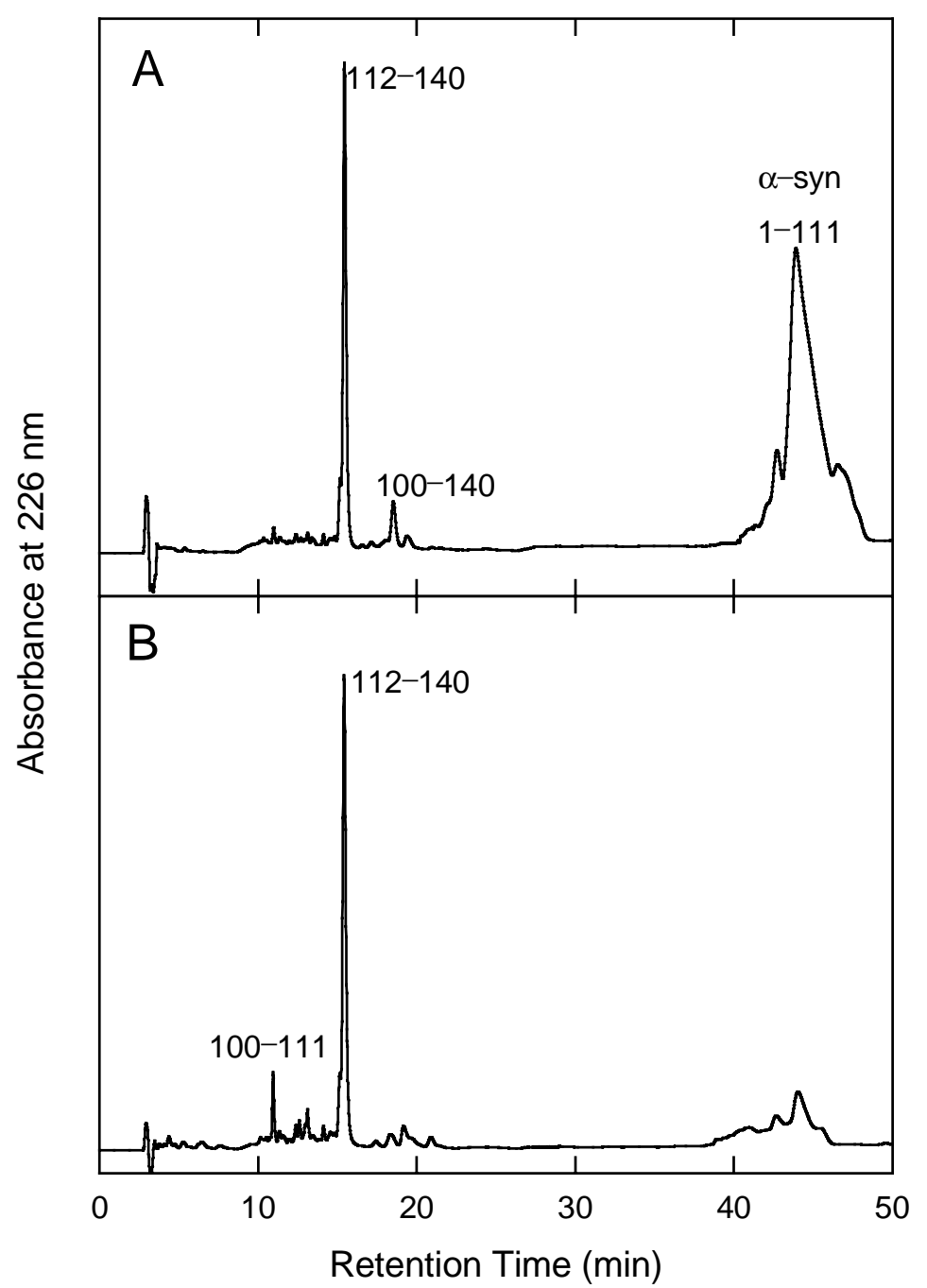

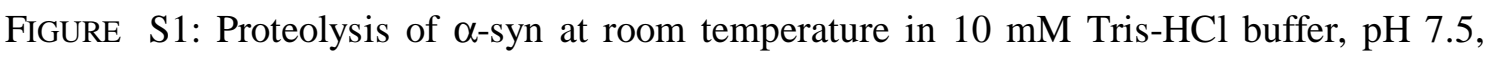
containing $10 \mathrm{mM}$ SDS with thermolysin. The $\alpha$-syn concentration was $0.6 \mathrm{mg} / \mathrm{mL}$ and the E/S ratio 1:100 (by weight). After 2 hours a second addition of thermolysin was added to the mixture and then, after 4 hours of incubation, an aliquot was taken from the mixture and analysed by RP-HPLC (A). Then, a third addition of thermolysin was made and, after additional incubation for 20 hours, an aliquot was again analysed by RP-HPLC (B). The chromatograms in A and B clearly show that the proteolysis of the 140-residue chain of $\alpha$-syn is rather selective and that fragment $112-140$ is the main C-terminal fragment, accompanied by the complementary $\mathrm{N}$-terminal fragment $1-111$, this last coeluting with intact $\alpha$-syn. Of interest, also the minor fragment $100-140$ is initially formed, that later on appears to be hydrolysed to the complementary fragments 100-111 and 112-140. 

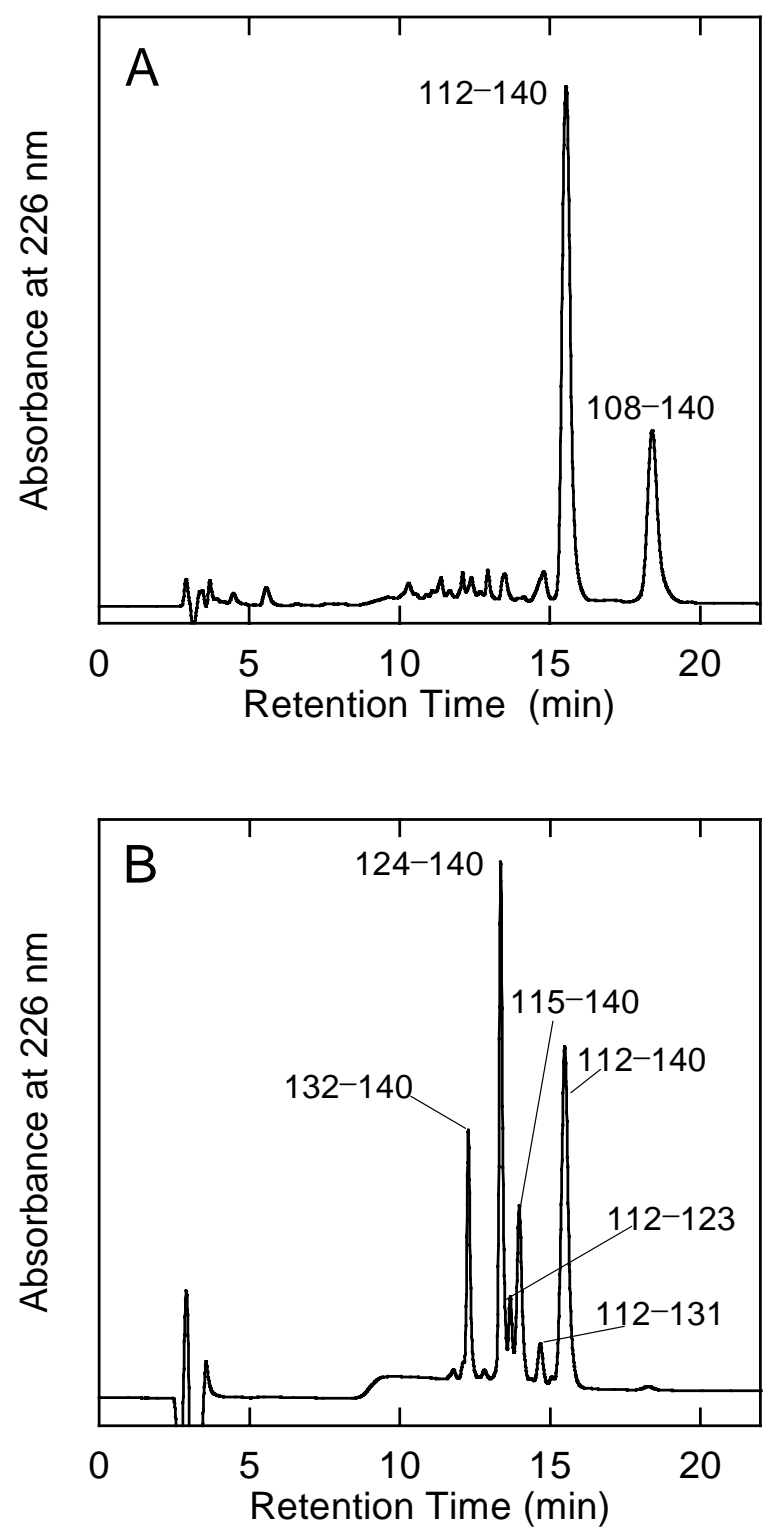

FIGURE S2: (A) Proteolysis of fragment 108-140 by thermolysin in the presence $10 \mathrm{mM}$ SDS. Proteolysis of fragment 108-140 was carried out using thermolysin as described under Experimental Procedures. (B) Fingerprinting analysis of the thermolytic fragment 112-140 contained in the chromatographic peak at RT 15.4 min of the RP-HPLC chromatogram shown in A. Fingerprinting by the Glu-specific V8-protease of peptide 112-140 was performed using an $\mathrm{E} / \mathrm{S}$ ratio of 1:50 (by weight) at a peptide concentration of $0.6 \mathrm{mg} / \mathrm{mL}$ in $10 \mathrm{mM}$ Tris-HCl, $\mathrm{pH}$ 7.5. The reaction mixtures were analyzed by RP-HPLC using a Vydac $\mathrm{C}_{18}$ column using the conditions described under Experimental Procedures. The peptides produced by V8protease digestion of fragment 112-140 are those expected for cleavages at Glu residues (see the amino acid sequence of $\alpha$-syn in Figure 1, main text). 
Table S2: Fingerprinting Analysis by Endoproteinase Glu-C (V8-protease) of Fragment 112-140.

\begin{tabular}{cccc}
\hline \multicolumn{4}{c}{ Molecular mass $(\mathrm{Da})$} \\
\hline $\mathrm{RT}(\mathrm{min})$ & observed $^{b}$ & calculated $^{c}$ & fragment \\
\hline 12.8 & 1071.5 & 1071.1 & $132-140$ \\
13.4 & 2007.1 & 2007.1 & $124-140$ \\
13.7 & 1386.0 & 1386.5 & $112-123$ \\
13.9 & 3021.2 & 3021.1 & $115-140$ \\
14.6 & 2324.2 & 2323.5 & $112-131$ \\
15.4 & 3376.4 & 3376.5 & $112-140$
\end{tabular}

${ }^{a}$ The molecular masses of the fragments have been determined by ESI-MS analysis of fragments isolated after RP-HPLC (see Figure S2). ${ }^{b}$ Determined by ESI-MS. ${ }^{c}$ Molecular masses calculated on the basis of the amino acid sequence of human $\alpha$-syn. 


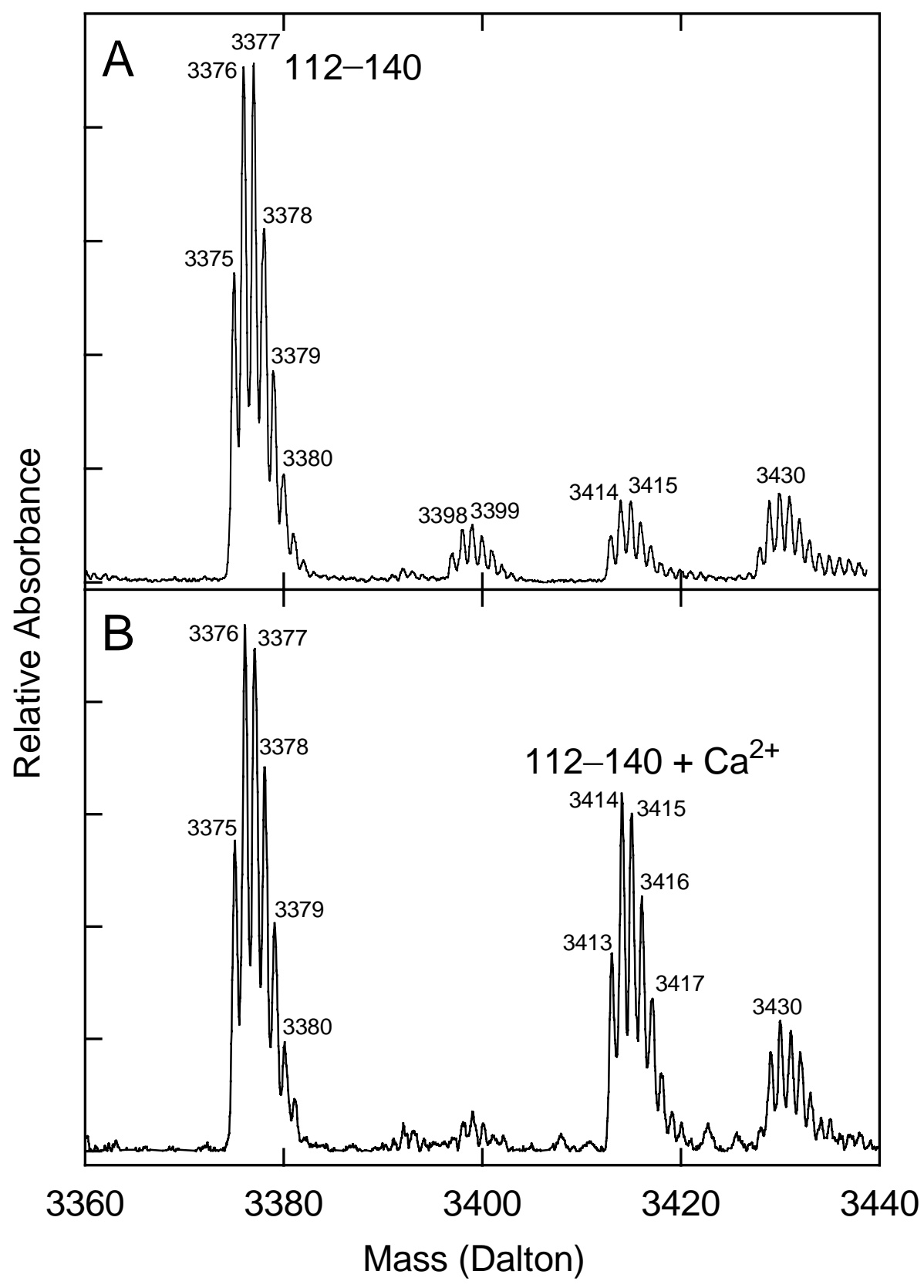

FIGURE S3: Deconvoluted ESI-MS spectra of peptide 112-140 (A) and of the same sample after addition of calcium (B). The peptide was treated with $1 \mathrm{mM}$ EGTA, purified by RPHPLC and dried. The peptide sample was dissolved in water and analyzed by ESI-MS (A). Another peptide sample was prepared in water containing $1 \mathrm{mM} \mathrm{CaCl}_{2}$ and then analyzed by ESI-MS (B). After this treatment, besides the MS signal (3376) of the peptide without any adduct, also a clear-cut increase of the MS signal (3414) corresponding to the +38 adduct (+ $\mathrm{Ca}$ ) of the peptide is observed. Mass spectra were acquired with a Micro Q-Tof mass spectrometer (Micromass, Manchester, UK). The measurements were conducted at a capillary voltage of 3-3.2 kV and a cone voltage of $40-45 \mathrm{~V}$. 